\title{
Association between the HOTATR polymorch Paper Association between the HOTAIR polymorphisms and cancer risk: an updated meta-analysis
}

\author{
Zhao-Xiong Zhang ${ }^{1}$, Xue Tong ${ }^{1}$, Wan-Ni Zhang ${ }^{1}$, Wei-Neng Fu ${ }^{1}$ \\ ${ }^{1}$ Department of Medical Genetics, China Medical University, Shenyang, 110122, P.R. China \\ Correspondence to: Wei-Neng Fu, email: wnfu@cmu.edu.cn
}

Keywords: HOTAIR, SNP, cancer, susceptible risk, meta-analysis

Received: July 15, $2016 \quad$ Accepted: December 01, 2016

Published: December 10, 2016

\section{ABSTRACT}

Purpose: LncRNA HOTAIR plays an important role in many cancer. Several studies have shown that some HOTAIR SNPs might be associated with tumor risk in casecontrol studies, but the results are inconsistent and inconclusive. Therefore, it is necessary to better evaluate association between the HOTAIR SNPs and the risk of cancer.

Results: rs920778, rs7958904 and rs874945 but not rs4759314 and rs1899663 loci were significantly related to cancer risk, among of which rs920778 and rs874945 increased and rs7958904 decreased cancer risk, respectively. Moreover, rs920778 is significantly susceptible in both Asian population and digestive cancer risks.

Materials and Methods: Data were collected from PubMed, Embase and Web of Science. A total of 11 case-control studies were selected for the quantitative analysis. Software Stata (Version 12) was used to calculate Odds ratios (ORs) and $\mathbf{9 5 \%}$ confidence intervals (CIs) to evaluate the strength of the associations. Subgroup analysis, sensitivity analysis, and publication bias were also performed. Five HOTAIR SNPs were finally enrolled in the study.

Conclusions: HOTAIR SNP rs920778, rs7958904 and rs874945 are susceptible to cancer risk. SNP rs920778 is also a useful risk factor in evaluation of Asian population and digestive cancer. In addition, the cancer risk SNP rs874945 is first reported in the meta-analysis.

\section{INTRODUCTION}

Long non coding RNAs (LncRNAs) are a class of regulatory RNAs that are longer than 200 nucleotides and lack protein coding capacity [1]. LncRNAs play critical roles in physiologic and pathologic processes, including carcinogenesis [2, 3]. In addition, IncRNAs are also involved in cellular processes such as differentiation [4], proliferation [5], apoptosis [6], metabolism [7] and autophagy [8].

Recently, genome-wide association studies (GWAS) have revealed a large number of genetic variants related to different types of cancer. For examples, Guo et al. identified 45 candidate lncRNAs regulated by noncoding SNPs in prostate cancer [9]. Yuan et al. found that a novel SNP rs114020893 in the lncRNA NEXN-AS1 gene is significantly associated with an increased risk of lung cancer [10]. However, there are some limitations in the GWAS. For examples, at least one-third of the identified variants in non-coding intervals regulatory regions modulate transcription factor binding $[11,12]$, but the relationship between phenotype-related loci and lncRNAs is largely unknown. Furthermore, many GWAS results have shown substantial heterogeneity in allele frequencies across different population [13]. Therefore, further study on a single candidate lncRNA gene in the development of cancer is necessary.

LncRNA HOTAIR, which is located within the Homeobox C (HOXC) gene cluster on chromosome 12 and is co-expressed with HOXC genes, is a inhibitor in the HOXD gene transcription [14]. As an oncogene, HOTAIR is overexpressed in many cancer and involved in cancer proliferation, migration, invasion, progression and poor prognosis, suggesting that it might be a potential novel target in cancer therapy $[15,16]$.

As a class of genetic variants, SNPs are widely used in prediction of disease risk [17], prognosis [18] and clinical outcome [19]. Recently, several studies have 
summarized the associations of HOTAIR SNPs with cancer risk. However, some results are controversial. For example, two groups reported that there is no association between rs4759314 polymorphism and cancer risk $[20,21]$, but Qi et al. found that rs4759314 is in association with cancer risk [22]. Obviously, the association analysis between HOTAIR SNPs and cancer susceptibility is still necessary.

In the study, a total of 5 SNPs collected from 11 articles were finally enrolled for meta-analysis, three of which were significantly associated with cancer risk, suggesting they are important cancer risk factors and potential targets in future clinical study.

\section{RESULTS}

\section{Characteristics of eligible studies}

A total of 26 articles on relationship between HOTAIR SNPs and cancer risk were retrieved after first search in PubMed, Embase and Web of Science. As shown in Figure 1, 11 case-control publications including 10867 patients and 13172 controls met the inclusion criteria and 5 HOTAIR SNPs were involved in the meta-analysis [23-33]. The main characteristics of 5 HOTAIR SNPs were listed in Table 1. Of the 11 studies, the HOTAIR rs4759314 in 8 studies [23-25, 27, 29-32], rs920778 in 6 studies [23, 26-28, 30, 33], rs7958904 in 4 studies $[24,29,31,32]$, rs874945 in 4 studies [24, 29, 31, 32] and rs1899663 in 3 studies [23, 27, 30] were analyzed, respectively. Genotyping data obtained came from PCRRFLP and TaqMan detection methods. Genotype and allele frequencies, sample size and other information were revealed in Table 1. Of the 11 studies, 4 presented a significant deviation from HWE (2 studies on rs4759314 $[31,32], 3$ on rs920778 [23, 27, 33] and 2 on rs874945 [31, 32]). NOS scale in each study was assessed and the results showed that all the studies had high-quality (Table 2). Of the 11 studies, 2 populations including Chinese and Turks and 7 types of cancer involving ESCC, colorectal cancer, gastric cancer, breast cancer, osteosarcoma, EOC and cervical cancer were used for group analysis (Table 1).

\section{Association between HOTAIR rs4759314 and cancer susceptibility}

We analyzed the association between rs4759314 $(A>G)$ and cancer susceptibility in 8 studies with 7025 cases and 8157 controls. As a result, we did not find a significant association between rs4759314 (A>G) and cancer susceptibility in any genetic model in general population. Moreover, we did not find a significant association between them in subgroup analysis of cancer type either (Table 3).

\section{Association between HOTAIR rs920778 and cancer susceptibility}

Association between rs920778 (C>T ) and cancer risk was analyzed in 6 studies with 3842 cases and 5015 controls. Overall, we observed a significantly increased risk of cancer susceptibility in homozygote comparison, dominant model and recessive model (TT versus CC: $\mathrm{OR}=1.675,95 \% \mathrm{CI} 1.034-2.714, \mathrm{P}_{\mathrm{h}}<0.01 ; \mathrm{CT} / \mathrm{TT}$ versus CC: $\mathrm{OR}=1.278,95 \%$ CI $1.025-1.593, \mathrm{P}_{\mathrm{h}}=0.10$; TT versus CC/CT: OR $=1.671,95 \%$ CI $1.195-2.337, \mathrm{P}_{\mathrm{h}}<$ 0.01 ), but not in allele contrast model and heterozygote comparison (Table 3). In subgroup analysis, rs920778 (C>T ) showed a significant increased risk of cancer in all the genetic models in Asian population (T versus $\mathrm{C}$ : $\mathrm{OR}=1.464,95 \%$ CI 1.362-1.574, $\mathrm{P}_{\mathrm{h}}=0.873$; TT versus CC: $\mathrm{OR}=2.647,95 \%$ CI 2.173-3.226, $\mathrm{P}_{\mathrm{h}}=0.452 ; \mathrm{CT}$ versus CC: $\mathrm{OR}=1.322,95 \%$ CI $1.197-1.459, \mathrm{P}_{\mathrm{h}}=0.988$; $\mathrm{CT} / \mathrm{TT}$ versus $\mathrm{CC}: \mathrm{OR}=1.468,95 \% \mathrm{CI} 1.336-1.613, \mathrm{P}_{\mathrm{h}}=$ 0.980 ; TT versus CC/CT: OR $=2.081,95 \%$ CI $1.523-$ $2.845, \mathrm{P}_{\mathrm{h}}=0.013$ ) (Figure 2A). In cancer type analysis, rs920778 $(\mathrm{C}>\mathrm{T})$ also revealed a significantly increased risk of digestive cancer in all the genetic models ( $\mathrm{T}$ versus $\mathrm{C}: \mathrm{OR}=1.367,95 \% \mathrm{CI} 1.165-1.605, \mathrm{P}_{\mathrm{h}}=0.054 ; \mathrm{TT}$ versus $C C:$ OR $=2.174,95 \%$ CI 1.261-3.748, $\mathrm{P}_{\mathrm{h}}=0.008$; CT versus $\mathrm{CC}$ : $\mathrm{OR}=1.314,95 \%$ CI 1.186-1.455, $\mathrm{P}_{\mathrm{h}}=$ 0.564 ; CT/TT versus CC: OR $=1.440,95 \%$ CI 1.294 $1.601, \mathrm{P}_{\mathrm{h}}=0.339$; $\mathrm{TT}$ versus $\mathrm{CC} / \mathrm{CT}: \mathrm{OR}=1.941,95 \% \mathrm{CI}$ $1.120-3.364, \mathrm{P}_{\mathrm{h}}=0.002$ ) (Figure 2B).

\section{Association between HOTAIR rs7958904, rs874945 or rs1899663 and cancer susceptibility}

Analysis results between HOTAIR rs7958904, rs 874945 or rs 1899663 and cancer risk were shown in Table 4. In general, there existed a significant association of rs7958904 polymorphism with decreased cancer risk in all genetic models (C versus $\mathrm{G}$ : $\mathrm{OR}=0.818,95 \% \mathrm{CI}$ 0.766-0.875, $\mathrm{P}_{\mathrm{h}}=0.313$; $\mathrm{CC}$ versus $\mathrm{GG}: \mathrm{OR}=0.641$, 95\% CI 0.544-0.755, $\mathrm{P}_{\mathrm{h}}=0.319$; GC versus GG: OR = $0.853,95 \%$ CI $0.782-0.931, \mathrm{P}_{\mathrm{h}}=0.754$; GC/CC versus GG: $\mathrm{OR}=0.814,95 \%$ CI $0.749-0.884, \mathrm{P}_{\mathrm{h}}=0.339 ; \mathrm{CC}$ versus $\mathrm{GG} / \mathrm{GC}$ : OR $=0.682,95 \%$ CI $0.582-0.801, \mathrm{P}_{\mathrm{h}}$ $=0.393$ ) (Figure 3A). When analyzing rs874945, we observed a significantly increased cancer risk in allele contrast model, homozygote comparison and dominant model (A versus G: OR = 1.106, 95\% CI 1.021-1.197, $\mathrm{P}_{\mathrm{h}}=0.816$; AA versus GG: OR $=1.259,95 \% \mathrm{CI} 1.015-$ $1.562, \mathrm{P}_{\mathrm{h}}=0.851 ; \mathrm{GA} / \mathrm{AA}$ versus $\mathrm{GG}$ : OR $=1.104,95 \%$ CI 1.005-1.212, $\left.\mathrm{P}_{\mathrm{h}}=0.896\right)$, but not in heterozygote comparison and recessive model (Figure 3B). Similar to rs4759314, rs1899663 polymorphism was not in significant association with cancer risk in all genetic models (Table 4). 
Table 1: Characteristics of studies included in the meta-analysis

\begin{tabular}{|c|c|c|c|c|c|c|c|c|c|c|c|c|c|c|c|c|}
\hline Author & Year & Country & Ethnicity & Cancer Type & $\begin{array}{c}\text { Source of } \\
\text { Control }\end{array}$ & $\begin{array}{c}\text { Genetyping } \\
\text { Method }\end{array}$ & Case/Control & & Case & & & Control & & $\begin{array}{l}\text { Risk to } \\
\text { cancer }\end{array}$ & $\begin{array}{l}\text { P for } \\
\text { HWE }\end{array}$ & Quality \\
\hline & & & & $\mathrm{rs} 4759314 \mathrm{~A}>\mathrm{G}$ & & & & AA & $\mathrm{AG}$ & GG & $\mathrm{AA}$ & $\mathrm{AG}$ & GG & & & \\
\hline Zhang & 2014 & China & Asian & $\mathrm{ESCC}$ & $\mathrm{HB}$ & PCR-PFLP & $1000 / 1000$ & 917 & 81 & 2 & 910 & 89 & 1 & none & 0.436 & 8 \\
\hline Xue & 2014 & China & Asian & Colorectal cancer & $\mathrm{HB}$ & TaqMan & $1733 / 1855$ & 1528 & 200 & 5 & 1608 & 236 & 11 & none & 0.467 & 7 \\
\hline Guo & 2015 & China & Asian & GCA & $\mathrm{HB}$ & PCR-PFLP & $515 / 654$ & 461 & 53 & 1 & 589 & 64 & 1 & none & 0.587 & 7 \\
\hline Pan & 2015 & China & Asian & Gastric cancer & $\mathrm{HB}$ & PCR-PFLP & $500 / 1000$ & 451 & 48 & 1 & 914 & 83 & 3 & none & 0.448 & 7 \\
\hline $\mathrm{Du}$ & 2015 & China & Asian & Gastric cancer & $\mathrm{HB}$ & TaqMan & $1275 / 1644$ & 1083 & 186 & 6 & 1464 & 172 & 8 & increased & 0.230 & 8 \\
\hline Yan & 2015 & China & Asian & Breast cancer & PB & PCR-PFLP & $502 / 504$ & 451 & 50 & 1 & 448 & 54 & 2 & none & 0.785 & 7 \\
\hline Zhou & 2016 & China & Asian & Osteosarcoma & $\mathrm{HB}$ & TaqMan & $500 / 500$ & 423 & 62 & 15 & 425 & 64 & 11 & none & $<0.05$ & 7 \\
\hline \multirow[t]{2}{*}{ Wu } & 2016 & China & Asian & EOC & HB & TaqMan & $1000 / 1000$ & 819 & 140 & 41 & 852 & 125 & 23 & increased & $<0.05$ & 8 \\
\hline & & & & rs920778 C>T & & & & $\mathrm{CC}$ & $\mathrm{CT}$ & TT & $\mathrm{CC}$ & $\mathrm{CT}$ & TT & & & \\
\hline Zhang & 2014 & China & Asian & ESCC & HB & PCR-RFLP & $2098 / 2150$ & 1091 & 826 & 181 & 1323 & 749 & 78 & increased & $<0.05$ & 8 \\
\hline Bayram & 2015 & Turkish & Caucasian & Breast cancer & $\mathrm{IIB}$ & TaqMan & $123 / 122$ & 31 & 52 & 40 & 15 & 66 & 41 & increased & 0.140 & 6 \\
\hline Pan & 2015 & China & Asian & Gastric cancer & $\mathrm{HB}$ & PCR-RFLP & $800 / 1600$ & 420 & 321 & 59 & 980 & 575 & 45 & increased & $<0.05$ & 7 \\
\hline Bayram & 2015 & Turkish & Caucasian & Gastric cancer & $\mathrm{HB}$ & TaqMan & $104 / 209$ & 20 & 52 & 32 & 38 & 105 & 66 & none & 0.738 & 6 \\
\hline Yan & 2015 & China & Asian & Breast cancer & $\mathrm{PB}$ & PCR-RFLP & $502 / 504$ & 12 & 151 & 339 & 18 & 190 & 296 & increased & 0.060 & 7 \\
\hline \multirow[t]{2}{*}{ Qiu } & 2016 & China & Asian & Cervical cancer & $\mathrm{HB}$ & TaqMan & $215 / 430$ & 90 & 78 & 47 & 226 & 150 & 54 & increased & $<0.05$ & 7 \\
\hline & & & & rs $7958904 \mathrm{G}>\mathrm{C}$ & & & & GG & $\mathrm{GC}$ & $\mathrm{CC}$ & GG & $\mathrm{GC}$ & $\mathrm{CC}$ & & & \\
\hline Xue & 2014 & China & Asian & Colorectal cancer & HB & TaqMan & $1731 / 1852$ & 1019 & 605 & 107 & 992 & 704 & 156 & decreased & 0.052 & 7 \\
\hline $\mathrm{Du}$ & 2015 & China & Asian & Gastric cancer & $\mathrm{HB}$ & TaqMan & $739 / 1057$ & 412 & 276 & 51 & 568 & 404 & 85 & none & 0.271 & 8 \\
\hline Zhou & 2016 & China & Asian & Osteosarcoma & $\mathrm{HB}$ & TaqMan & $900 / 900$ & 524 & 320 & 56 & 466 & 346 & 88 & decreased & $<0.05$ & 7 \\
\hline \multirow[t]{2}{*}{ Wu } & 2016 & China & Asian & EOC & $\mathrm{HB}$ & TaqMan & $1000 / 1000$ & 594 & 355 & 51 & 533 & 380 & 87 & decreased & 0.105 & 8 \\
\hline & & & & $\mathrm{rs} 874945 \mathrm{G}>\mathrm{A}$ & & & & GG & GA & $\mathrm{AA}$ & GG & GA & $\mathrm{AA}$ & & & \\
\hline Xue & 2014 & China & Asian & Colorectal cancer & $\mathrm{HB}$ & TaqMan & $1147 / 1202$ & 751 & 356 & 40 & 817 & 346 & 39 & none & 0.749 & 7 \\
\hline $\mathrm{Du}$ & 2015 & China & Asian & Gastric cancer & $\mathrm{HB}$ & TaqMan & $751 / 1057$ & 495 & 225 & 31 & 714 & 307 & 36 & none & 0.672 & 8 \\
\hline Zhou & 2016 & China & Asian & Osteosarcoma & $\mathrm{HB}$ & TaqMan & $900 / 900$ & 577 & 256 & 67 & 608 & 243 & 49 & increased & $<0.05$ & 7 \\
\hline \multirow[t]{2}{*}{ Wu } & 2016 & China & Asian & EOC & $\mathrm{HB}$ & TaqMan & $1000 / 1000$ & 665 & 283 & 52 & 677 & 279 & 44 & none & $<0.05$ & 8 \\
\hline & & & & rs $1899663 \mathrm{G}>\mathrm{T}$ & & & & GG & GA & $\mathrm{AA}$ & GG & GA & $\mathrm{AA}$ & & & \\
\hline Zhang & 2014 & China & Asian & ESCC & $\mathrm{HB}$ & PCR-RFLP & $1000 / 1000$ & 725 & 256 & 19 & 724 & 250 & 26 & none & 0.430 & 7 \\
\hline Pan & 2015 & China & Asian & Gastric cancer & $\mathrm{HB}$ & PCR-RFLP & $500 / 1000$ & 376 & 118 & 6 & 732 & 255 & 13 & none & 0.078 & 7 \\
\hline Yan & 2015 & China & Asian & Breast cancer & $\mathrm{PB}$ & PCR-RFLP & $502 / 504$ & 339 & 149 & 14 & 326 & 158 & 20 & none & 0.876 & 7 \\
\hline
\end{tabular}

ESCC: Esophageal squamous cell carcinoma; GCA: Gastric cardia adenocarcinoma; EOC: Epithelial ovarian cancer; HB: Hospital-based; PB: Population-based; HWE: Hardy-Weinberg equilibrium.

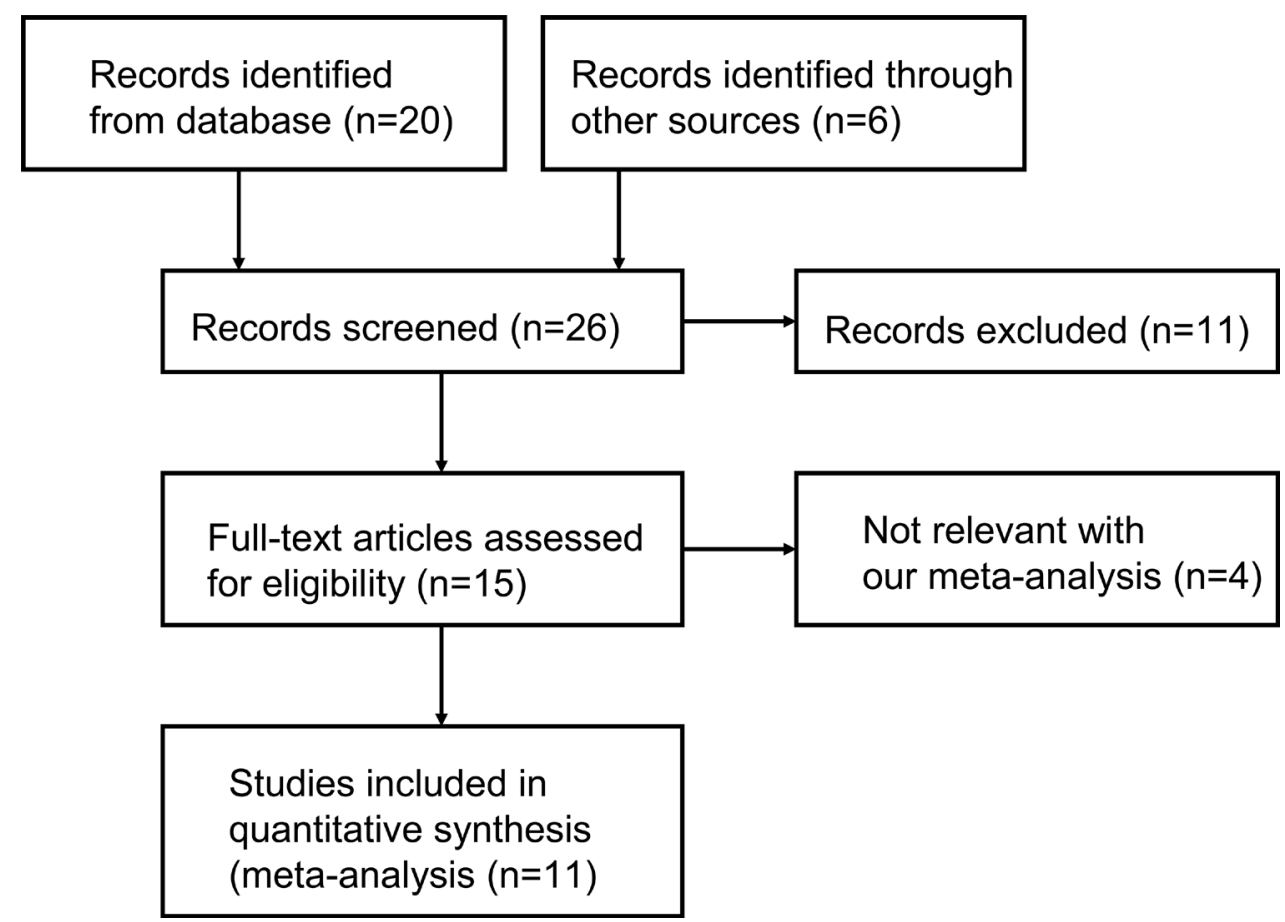

Figure 1: Flow diagram summarizing the selection of eligible studies. 
Table 2: Newcastle-ottawa quality assessment scale for each included study

\begin{tabular}{|c|c|c|c|c|c|c|c|c|c|c|}
\hline \multirow[b]{2}{*}{ Studies } & \multicolumn{4}{|c|}{ Selection } & \multicolumn{2}{|c|}{ Comparability } & \multicolumn{3}{|c|}{ Exposure } & \multirow[b]{2}{*}{$\begin{array}{c}\text { Total } \\
\text { quality } \\
\text { score }\end{array}$} \\
\hline & $\begin{array}{c}\text { Case } \\
\text { definition } \\
\text { adequate }\end{array}$ & $\begin{array}{l}\text { Representativeness } \\
\text { of the cases }\end{array}$ & $\begin{array}{c}\text { Selection } \\
\text { of } \\
\text { controls }\end{array}$ & $\begin{array}{c}\text { Definition } \\
\text { of controls }\end{array}$ & $\begin{array}{l}\text { Adjustment } \\
\text { for age }\end{array}$ & $\begin{array}{c}\text { Adjustment } \\
\text { for lifestyle/ } \\
\text { traditional } \\
\text { risk factors }\end{array}$ & $\begin{array}{c}\text { Ascertainment } \\
\text { of exposure }\end{array}$ & $\begin{array}{c}\text { Uniform } \\
\text { method of } \\
\text { ascertainment }\end{array}$ & $\begin{array}{l}\text { Non- } \\
\text { response } \\
\text { rate }\end{array}$ & \\
\hline $\begin{array}{l}\text { Zhang } \\
2014\end{array}$ & 1 & 1 & 1 & 1 & 1 & 1 & 0 & 1 & 1 & 8 \\
\hline Xue 2014 & 1 & 1 & 1 & 1 & 1 & 1 & 0 & 1 & 0 & 7 \\
\hline Guo 2015 & 1 & 1 & 1 & 1 & 1 & 1 & 0 & 1 & 0 & 7 \\
\hline $\begin{array}{l}\text { Bayram } \\
2015\end{array}$ & 1 & 1 & 0 & 1 & 1 & 1 & 0 & 1 & 0 & 6 \\
\hline Pan 2015 & 1 & 1 & 1 & 1 & 1 & 1 & 0 & 1 & 0 & 7 \\
\hline $\begin{array}{l}\text { Bayram } \\
2015\end{array}$ & 1 & 1 & 0 & 1 & 1 & 1 & 0 & 1 & 0 & 6 \\
\hline Du 2015 & 1 & 1 & 1 & 1 & 1 & 1 & 0 & 1 & 1 & 8 \\
\hline Yan 2015 & 1 & 1 & 1 & 1 & 1 & 1 & 0 & 1 & 0 & 7 \\
\hline Zhou 2016 & 1 & 1 & 1 & 1 & 1 & 1 & 0 & 1 & 0 & 7 \\
\hline Wu 2016 & 1 & 1 & 1 & 1 & 1 & 1 & 0 & 1 & 1 & 8 \\
\hline Qiu 2016 & 1 & 1 & 1 & 1 & 1 & 1 & 0 & 1 & 0 & 7 \\
\hline
\end{tabular}

Table 3: Results of meta-analysis for rs4759314 and rs920778 polymorphisms and the risk of cancer

\begin{tabular}{|c|c|c|c|c|c|c|c|c|c|c|c|c|c|c|c|c|}
\hline Locus & $\mathrm{N}$ & $\mathrm{OR}(95 \% \mathrm{CI})$ & $\mathrm{P}_{\mathrm{h}}$ & $I^{2}(\%)$ & OR $(95 \% \mathrm{CI})$ & $\mathrm{P}_{\mathrm{h}}$ & $I^{2}(\%)$ & $\mathrm{OR}(95 \% \mathrm{CI})$ & $\mathrm{P}_{\mathrm{h}}$ & $I^{2}(\%)$ & $\mathrm{OR}(95 \% \mathrm{CI})$ & $\mathrm{P}_{\mathrm{h}}$ & $I^{2}(\%)$ & $\mathrm{OR}(95 \% \mathrm{CI})$ & $\mathrm{P}_{\mathrm{h}}$ & $I^{2}(\%)$ \\
\hline $\mathrm{rs} 4759314 \mathrm{~A}>\mathrm{G}$ & & GVS A & & & GG VS AA & & & AG VS AA & & & $\mathrm{AG} / \mathrm{GG}$ VS AA & & & GGVSAA/AG & & \\
\hline Overall & 8 & $1.084(0.933-1.259)$ & 0.018 & 58.5 & $1.298(0.916-1.840)$ & 0.483 & 0.0 & $1.067(0.922-1.235)$ & 0.065 & 47.4 & $1.080(0.929-1.225)$ & 0.036 & 53.4 & $1.283(0.906-1.817)$ & 0.504 & 0.0 \\
\hline \multicolumn{17}{|l|}{ Cancer Types } \\
\hline Digestive cancer & 5 & $1.060(0.861-1.305)$ & 0.018 & 66.5 & $0.771(0.403-1.473)$ & 0.778 & 0.0 & $1.080(0.866-1.348)$ & 0.017 & 66.9 & $1.072(0.860-1.337)$ & 0.015 & 67.6 & $0.761(0.398-1.456)$ & 0.789 & 0.0 \\
\hline Other cancer & 3 & $1.135(0.904-1.427)$ & 0.160 & 45.4 & $1.625(1.064-2.482)$ & 0.506 & 0.0 & $1.058(0.876-1.277)$ & 0.555 & 0.0 & $1.115(0.915-1.359)$ & 0.300 & 16.9 & $1.605(1.051-2.450)$ & 0.531 & 0.0 \\
\hline rs920778 C>T & & T VS C & & & TT VS CC & & & CT VS CC & & & $\mathrm{CT} / \mathrm{TT}$ VS CC & & & TT VS CC/CT & & \\
\hline Overall & 6 & $1.293(1.105-1.512)$ & 0.002 & 73.0 & $1.675(1.034-2.714)$ & 0.000 & 82.2 & $1.167(0.946-1.440)$ & 0.029 & 59.8 & $1.278(1.025-1.593)$ & 0.010 & 67.1 & $1.671(1.195-2.337)$ & 0.000 & 79.2 \\
\hline \multicolumn{17}{|l|}{ Ethnicity } \\
\hline Asian & 4 & $1.464(1.362-1.574)$ & 0.873 & 0.0 & $2.647(2.173-3.226)$ & 0.452 & 0.0 & $1.322(1.197-1.459)$ & 0.988 & 0.0 & $1.468(1.336-1.613)$ & 0.980 & 0.0 & $2.081(1.523-2.845)$ & 0.013 & 72.3 \\
\hline Caucasian & 2 & $0.858(0.671-1.096)$ & 0.321 & 0.0 & $0.672(0.349-1.293)$ & 0.199 & 39.4 & $0.608(0.251-1.474)$ & 0.064 & 70.8 & $0.633(0.287-1.397)$ & 0.080 & 67.4 & $0.958(0.663-1.383)$ & 0.976 & 0.0 \\
\hline \multicolumn{17}{|l|}{ Cancer Types } \\
\hline Digestive cancer & 3 & $1.367(1.165-1.605)$ & 0.054 & 65.8 & $2.174(1.261-3.748)$ & 0.008 & 79.4 & $1.314(1.186-1.455)$ & 0.564 & 0.0 & $1.440(1.294-1.601)$ & 0.339 & 7.6 & $1.941(1.120-3.364)$ & 0.002 & 83.7 \\
\hline Other cancer & 3 & $1.199(0.831-1.731)$ & 0.004 & 82.4 & $1.248(0.505-3.084)$ & 0.003 & 82.9 & $0.868(0.411-1.834)$ & 0.010 & 78.2 & $1.012(0.451-2.271)$ & 0.003 & 83.2 & $1.440(1.035-2.002)$ & 0.123 & 52.3 \\
\hline
\end{tabular}

\section{Sensitivity analysis result}

Sensitivity analysis was used to evaluate individual study's influence on the pooled results by deleting one single study each time from pooled analysis. As a result, rs920778 in Bayram's study [28] had a significant effect on the pooled OR. When this study was excluded in the genotype comparison of TT versus CC, the heterogeneity test was significantly reduced (data not shown).

\section{Publication bias analysis result}

A funnel plot was generated to assess potential publication bias. As shown in Figure 4, there was no any evidence of publication bias in rs4759314, rs7958904 or rs 874945 or rs 1899663 analysis. Result of publication bias was not shown. There was only slight statistical evidence of publication bias in rs920778 analysis $\left(P_{\mathrm{b}}=0.024\right.$, $\left.P_{\mathrm{e}}=0.040\right)$.

\section{DISCUSSION}

In the study, five potential HOTAIR SNPs including rs4759314, rs920778, rs7958904, rs874945 and rs 1899663 susceptible to cancer were identified and used for metaanalysis. SNP rs920778, rs4759314 and rs1899663 are located in the introns, rs7958904 in the coding region and rs 874945 is close to $3^{\prime}$ region of the HOTAIR gene, respectively [24].

Overall, our results provide evidences that rs 920778 , rs7958904 and rs 874945 but not rs4759314 and rs 1899663 loci are related to cancer risk, among of which rs920778 
A

Study

$\%$

ID

OR $(95 \% \mathrm{Cl}) \quad$ Weight

Asian

Zhang (2014)

Pan (2015)

Yan (2015)

Qiu (2016)

Subtotal $(I-$ squared $=0.0 \%, p=0.452$ )

Caucasian

Bayram (2015)

Bayram (2015)

Subtotal $(\mathrm{I}$-squared $=39.4 \%, \mathrm{p}=0.19 \%$

Overall (I-squared $=82.2 \%, p=0.000)$

NOTE: Weights are from random effects analysis

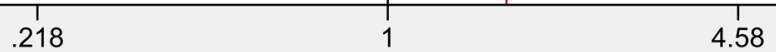

B

Study

$\%$
OR $(95 \% \mathrm{Cl}) \quad$ Weight

ID

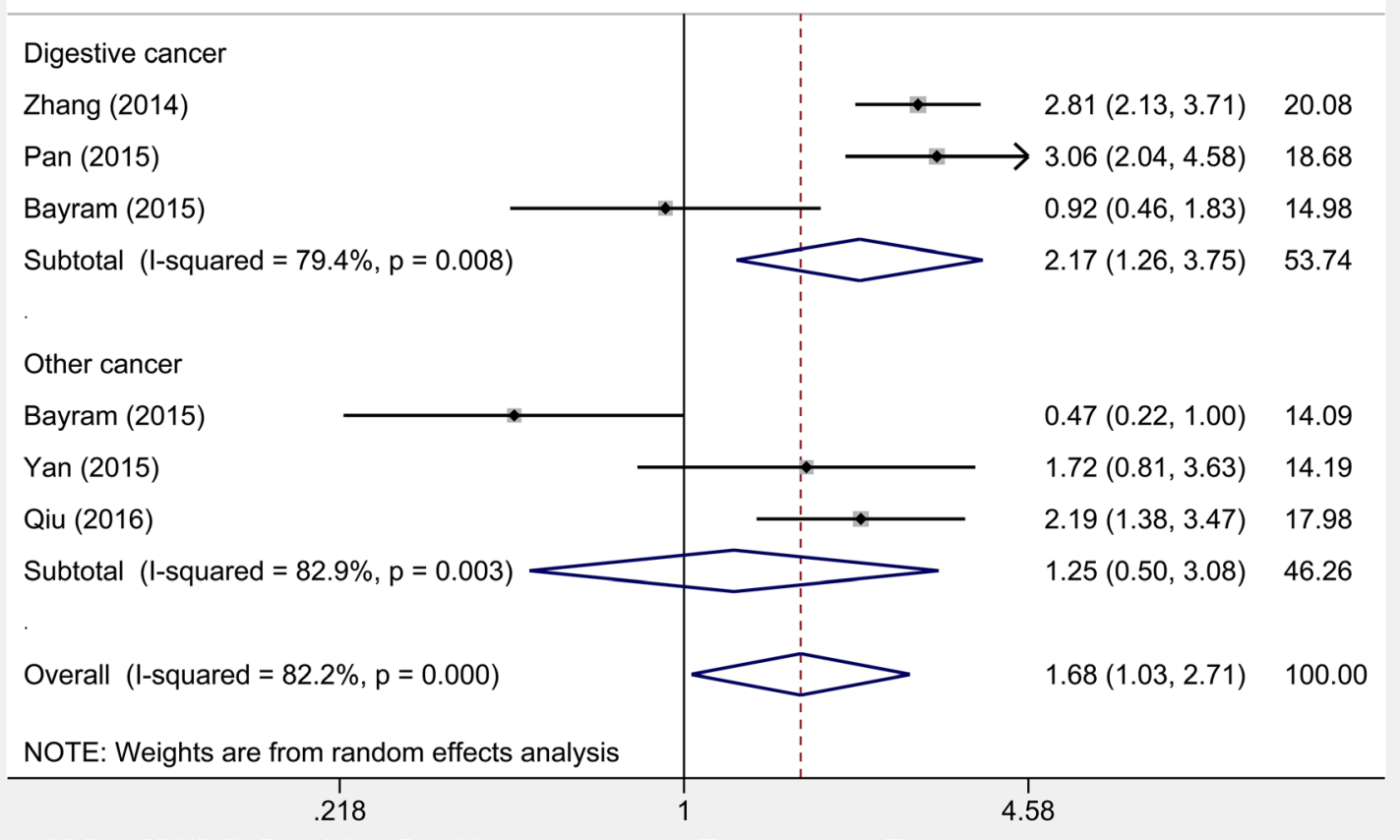

Figure 2: Subgroup analysis on relationship between HOTAIR rs920778 (C>T ) and cancer risk. (A) Ethnicity analysis. (B) Cancer type analysis. 
Table 4: Results of meta-analysis for rs7958904, rs874945 and rs1899663 polymorphisms and the risk of cancer

\begin{tabular}{|c|c|c|c|c|c|c|c|c|c|c|c|c|c|c|c|c|}
\hline Locus & $\mathrm{N}$ & $\mathrm{OR}(95 \% \mathrm{CI})$ & $\mathrm{P}_{\mathrm{h}}$ & $I^{2}(\%)$ & OR $(95 \% \mathrm{CI})$ & $\mathrm{P}_{\mathrm{h}}$ & $I^{2}(\%)$ & OR $(95 \% \mathrm{CI})$ & $\mathrm{P}_{\mathrm{h}}$ & $I^{2}(\%)$ & OR( $(95 \% \mathrm{CI})$ & $P_{h}$ & $I^{2}(\%)$ & $\mathrm{OR}(95 \% \mathrm{CI})$ & $\mathrm{P}_{\mathrm{h}}$ & $I^{2}(\%)$ \\
\hline rs7958904 G>C & & C VS G & & & CC VS GG & & & GC VS GG & & & $\mathrm{GC} / \mathrm{CC}$ VS GG & & & $\mathrm{CC} \mathrm{VS} \mathrm{GG/GC}$ & & \\
\hline Overall & 4 & $0.818(0.766-0.875)$ & 0.313 & 15.8 & $0.641(0.544-0.755)$ & 0.319 & 14.7 & $0.853(0.782-0.931)$ & 0.754 & 0.0 & $0.814(0.749-0.884)$ & 0.523 & 0.0 & $0.682(0.582-0.801)$ & 0.393 & 0.0 \\
\hline rs $874945 G>A$ & & A VS G & & & AA VS GG & & & GA VS GG & & & GA/AA VS GG & & & $\mathrm{AA}$ VS GG/GA & & \\
\hline Overall & 4 & $1.106(1.021-1.197)$ & 0.816 & 0.0 & $1.259(1.015-1.562)$ & 0.851 & 0.0 & $1.081(0.980-1.193)$ & 0.927 & 0.0 & $1.104(1.005-1.212)$ & 0.896 & 0.0 & $1.231(0.944-1.523)$ & 0.853 & 0.0 \\
\hline $\mathrm{rs} 1899663 \mathrm{G}>\mathrm{T}$ & & T VS G & & & TT VS GG & & & GT VS GG & & & GT/TT VS GG & & & TT VS GG/GT & & \\
\hline Overall & 3 & $0.928(0.825-1.043)$ & 0.774 & 0.0 & $0.737(0.487-1.114)$ & 0.894 & 0.0 & $0.956(0.834-1.095)$ & 0.675 & 0.0 & $0.937(0.821-1.069)$ & 0.714 & 0.0 & $0.746(0.494-1.125)$ & 0.891 & 0.0 \\
\hline
\end{tabular}

$\mathbf{N}$ : the number of studies; $\mathbf{P}_{\mathrm{h}}$ : P- value for heterogeneity test.

A

Study

$\%$

ID

OR $(95 \% \mathrm{Cl}) \quad$ Weight

Xue (2014)

Du (2015)

Zhou (2016)

Wu (2016)

Overall (I-squared $=14.7 \%, p=0.319$ )

Overall $(1$-squared $=14.7 \%, \mathrm{p}=0.319)$

$0.67(0.51,0.87) \quad 38.24$

$0.83(0.57,1.20) \quad 17.17$

$0.57(0.40,0.81) \quad 22.24$

$0.53(0.37,0.76) \quad 22.35$

$0.64(0.54,0.75) \quad 100.00$

.365

2.74

B

Study

$\%$

ID

OR $(95 \% \mathrm{Cl}) \quad$ Weight

\begin{tabular}{|c|c|c|}
\hline Xue (2014) & $1.12(0.71,1.75)$ & 24.09 \\
\hline Du (2015) & $1.24(0.76,2.04)$ & 18.92 \\
\hline Zhou (2016) & $1.44(0.98,2.12)$ & 29.44 \\
\hline Wu (2016) & $1.20(0.79,1.82)$ & 27.56 \\
\hline Overall (I-squared $=0.0 \%, p=0.851$ ) & $1.26(1.02,1.56)$ & 100.00 \\
\hline
\end{tabular}

Figure 3: Forest plot of the association between rs7958904 or rs874945 and cancer risk in a homozygote comparison model. (A) rs7958904 analysis result. (B) rs874945 analysis result. 
Table 5: The characteristics and comparison of different meta-analysis

\begin{tabular}{|c|c|c|c|c|c|c|c|}
\hline $\begin{array}{c}\text { Author } \\
\text { (Other meta } \\
\text { analysis) }\end{array}$ & $\begin{array}{l}\text { Enrolled } \\
\text { papers }\end{array}$ & $\begin{array}{c}\text { Case/ } \\
\text { Control }\end{array}$ & genetic model & enrolled SNPs & $\begin{array}{l}\text { overall risk } \\
\text { to cancer }\end{array}$ & $\begin{array}{c}\text { subgroup } \\
\text { (ethnicity) }\end{array}$ & $\begin{array}{c}\text { subgroup } \\
\text { (cancer type) }\end{array}$ \\
\hline \multirow[t]{3}{*}{ Tian } & 8 & $7151 / 8740$ & $\begin{array}{l}\text { three models: heterozygote comparison, homozygote comparison, } \\
\text { dominant model }\end{array}$ & rs920778 & increased risk & Asian & digestive cancer \\
\hline & & & One model: dominant model & rs4759314 & none & none & none \\
\hline & & & One model: dominant model & rs1899663 & none & none & none \\
\hline \multirow[t]{3}{*}{ Zhang } & 6 & & $\begin{array}{l}\text { five models: allele contrast model, dominant model, recessive } \\
\text { model, heterozygote comparison, homozygote comparison }\end{array}$ & rs920778 & increased risk & Asian & none \\
\hline & & & $\begin{array}{l}\text { five models: allele contrast model, dominant model, recessive } \\
\text { model, heterozygote comparison, homozygote comparison }\end{array}$ & rs4759314 & none & none & none \\
\hline & & & $\begin{array}{l}\text { five models: allele contrast model, dominant model, recessive } \\
\text { model, heterozygote comparison, homozygote comparison }\end{array}$ & rs 1899663 & none & none & none \\
\hline \multirow[t]{2}{*}{ Qi } & 9 & $7772 / 9075$ & $\begin{array}{l}\text { five models: allele contrast model, dominant model, recessive } \\
\text { model, heterozygote comparison, homozygote comparison }\end{array}$ & rs920778 & increased risk & Asian & gastric cancer \\
\hline & & & $\begin{array}{l}\text { five models: allele contrast model, dominant model, recessive } \\
\text { model, heterozygote comparison, homozygote comparison }\end{array}$ & rs4759314 & none & none & none \\
\hline \multirow[t]{4}{*}{ Zhang } & 8 & $7151 / 8740$ & $\begin{array}{l}\text { four models: allele contrast model, dominant model, recessive } \\
\text { model, homozygote comparison }\end{array}$ & rs920778 & increased risk & Asian & gastric cancer \\
\hline & & & $\begin{array}{l}\text { four models: allele contrast model, dominant model, recessive } \\
\text { model, homozygote comparison }\end{array}$ & rs4759314 & none & none & none \\
\hline & & & $\begin{array}{l}\text { four models: allele contrast model, dominant model, recessive } \\
\text { model, homozygote comparison }\end{array}$ & rs1899663 & none & none & none \\
\hline & & & $\begin{array}{l}\text { four models: allele contrast model, dominant model, recessive } \\
\text { model, homozygote comparison }\end{array}$ & rs7958904 & $\begin{array}{l}\text { decreased } \\
\text { risk }\end{array}$ & none & none \\
\hline \multirow[t]{5}{*}{ Present study } & 11 & $10867 / 13172$ & $\begin{array}{l}\text { five models: allele contrast model, dominant model, recessive } \\
\text { model, heterozygote comparison, homozygote comparison }\end{array}$ & rs920778 & increased risk & Asian & digestive cancer \\
\hline & & & $\begin{array}{l}\text { five models: allele contrast model, dominant model, recessive } \\
\text { model, heterozygote comparison, homozygote comparison }\end{array}$ & rs4759314 & none & none & none \\
\hline & & & $\begin{array}{l}\text { five models: allele contrast model, dominant model, recessive } \\
\text { model, heterozygote comparison, homozygote comparison }\end{array}$ & rs1899663 & none & none & none \\
\hline & & & $\begin{array}{l}\text { five models: allele contrast model, dominant model, recessive } \\
\text { model, heterozygote comparison, homozygote comparison }\end{array}$ & rs7958904 & $\begin{array}{l}\text { decreased } \\
\text { risk }\end{array}$ & none & none \\
\hline & & & $\begin{array}{l}\text { five models: allele contrast model, dominant model, recessive } \\
\text { model, heterozygote comparison, homozygote comparison }\end{array}$ & rs874945 & increased risk & none & none \\
\hline
\end{tabular}

and rs874945 increase and rs7958904 decreases cancer risk, respectively. In the study, we also found that rs920778 is susceptible to cancer in Asian population but not in Turks, which is in accord with Tian's study [20]. We speculate that there are two reasons to explain the difference. First, different genetic backgrounds may contribute to divergence because the distribution of HOTAIR allele frequency varies among Asians and Caucasians. Second, different populations have various lifestyles and are influenced by different environmental factors.

Previously, four papers have discussed the association of HOTAIR polymorphisms with cancer risk by meta-analysis [20-22, 34]. In comparison with these studies, our meta-analysis has the following characteristics (Table 5). In the study, we have selected 11 related articles more than that in any study mentioned above. The enrolled SNPs and cases including patients and controls in our study are also much more than those in any study, implying that our assessment of the relationship between the HOTAIR gene SNPs and cancer risk is relatively more precise. As for rs920778 polymorphism, all five meta-analysis studies including ours have same results which indicate that the polymorphism can increase cancer risk, especially in Asian population and digestive cancer. However, Bayram et al. did not find association of rs 920778 to gastric cancer in a Turkish population by a case-control study [28]. For SNP rs4759314, all five meta-analysis studies showed that the SNP is not a risk factor of cancer. For rs 1899663 , our study together with other three studies also obtained the negative association of the polymorphic site to cancer risk. Same to Zhang's group study [21] , our group study showed that rs7958904 site decreases cancer risk. In addition, we first analyzed the association of rs874945 to cancer risk. As a result, the polymorphic site decreases the risk of cancer.

Zhang et al. identified a intronic enhancer between $+1719 \mathrm{bp}$ and $+2353 \mathrm{bp}$ from the transcriptional start site of HOTAIR gene and the SNP rs920778 is just located in the region. Moreover, they found that the SNP can increase HOTAIR RNA expression by altering the enhancer activity in esophageal squamous cell carcinoma [23]. As a SNP in the coding sequence, rs7958904 does not alter HOTAIR transcript, but it is predicted to change the secondary structure of HOTAIR, indicating that the SNP deregulates HOTAIR expression by affecting the gene structure, leading to carcinogenesis [24]. SNP rs874945 is near $3^{\prime}$ region of the HOTAIR gene. It is well known that gene 
$3^{\prime}$ region is a regulatory target of coding gene products [35] and non-coding genes such as microRNA [36]. It has been reported that SNP rs1126579 in the CXCR2 gene $3^{\prime}$ region disrupts the binding site for miR-516a-3p, lead to a moderate increase in CXCR2 mRNA and protein expression, and increased MAPK signaling [37]. Even though SNP rs874945 functional study is not reported, we speculate that it functions by interfering the bind with other coding gene products and non-coding genes.

However, there are several limitations to the present study. Few studies were included in this metaanalysis, and this small sample size limits the power to detect the associations. Because the power of funnel plots, Egger's and Begg's test of publication bias may also greatly constrain our analysis, our conclusions should be interpreted cautiously. For examples, there was a slight publication bias in the studies on rs920778. We think that only English language studies selected in the study partially contributes the bias. In addition, subgroup analyses of the rs7958904, rs874945 and rs1899663 loci were not performed because the sample size in each study was relatively not large enough.

Although HOTAIR polymorphisms are associated with assessment of cancer risk, abnormal expression of HOTAIR itself is also useful in predicting cancer
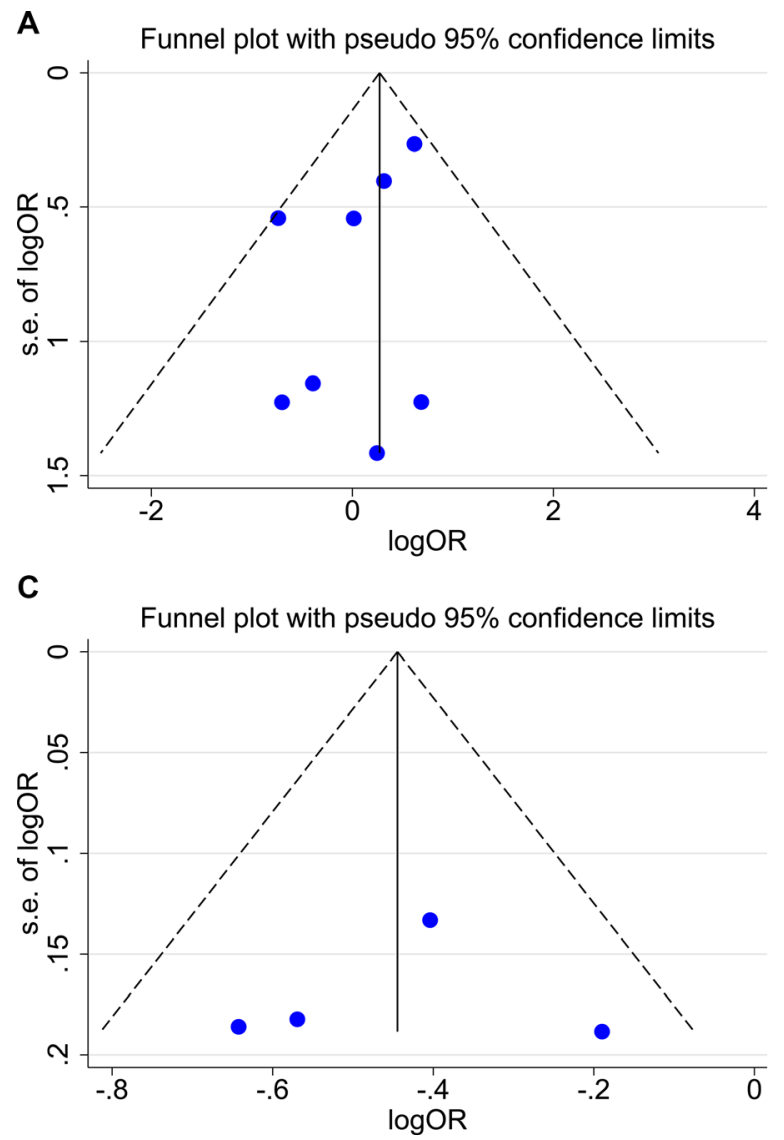

prognosis. For examples, high HOTAIR level is correlated to poor prognosis in digestive system tumors [38], estrogen-dependent malignant tumors [39], breast cancer, non-small cell lung cancer, laryngeal carcinoma [40], diffuse large B cell lymphoma [41], bladder transitional cell carcinoma [42] and acute leukemia [43]. Thus, HOTAIR is a key LncRNA in genesis and development of cancer.

In conclusion, HOTAIR SNP rs920778, rs7958904 and rs874945 are cancer risk factors, among of which rs920778 is also useful in evaluating cancer risk of Asian population and digestive cancer. Moreover, rs874945 as a cancer risk SNP is first reported in the meta-analysis.

\section{MATERIALS AND METHODS}

\section{Identification of eligible studies and data extraction}

We performed a publication search using PubMed, Embase and Web of Science updated on June 29,2016. The following search terms were used: "HOTAIR OR HOX transcript antisense intergenic RNA","polymorphism OR variation OR variant OR mutation", "cancer OR carcinoma OR tumor OR tumour OR neoplasm", respectively.
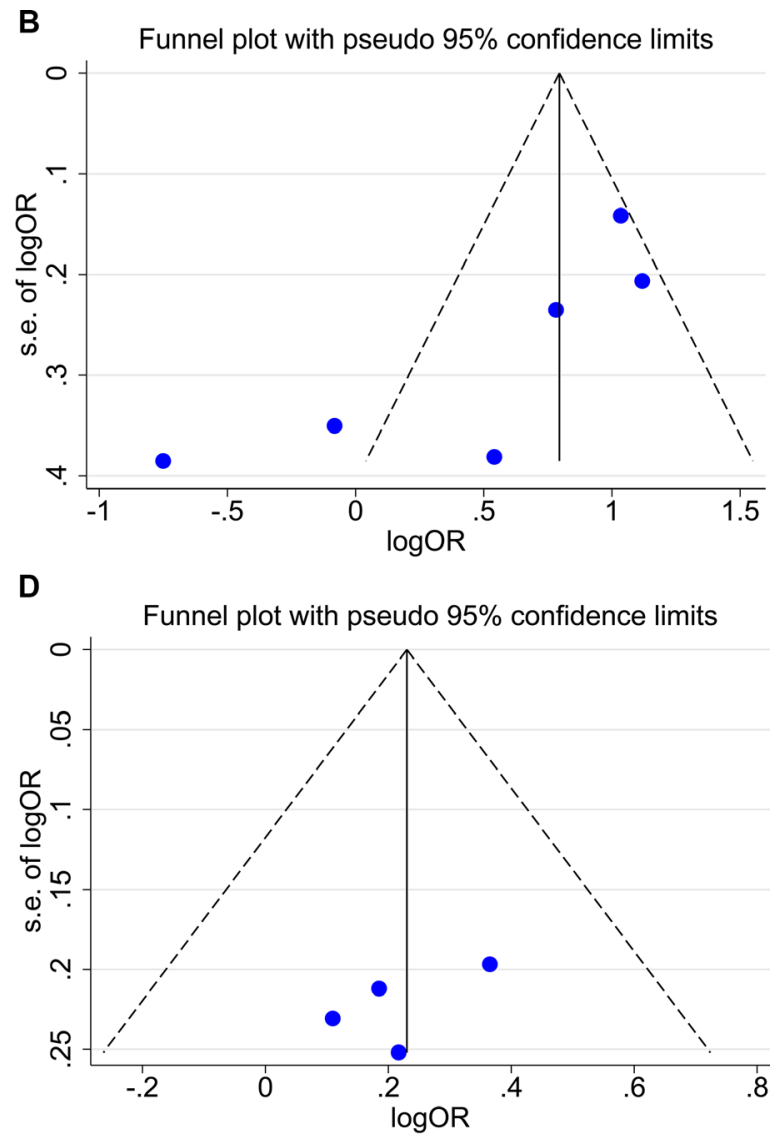

Figure 4: Funnel plot for publication bias test. (A) rs4759314 analysis result. (B) rs920778 analysis result. (C) rs795904 analysis result. (D) rs874945 analysis result. 
Searching was done without restriction on language or publication years. Inclusion criteria for studies were as followings: (1) articles on HOTAIR polymorphisms and cancer risk; (2) case-control studies; (3) studies that had detailed genotype frequency of cases and controls or could be calculated from the paper text. Exclusion criteria included: (1) abstract, comment, review; (2) duplication of the previous publications; (3) lack of usable genotype frequency data.

\section{Data extraction}

Based on the inclusion criteria, two reviewers (ZX Zhang and $\mathrm{WN} \mathrm{Fu}$ ) independently extracted information from all eligible publications. The following information were included in each study: name of first author, year of publication, country, ethnicity, cancer types, source of control, genotyping method, numbers of cases and controls, allele as well as genotype frequencies for cases and controls, $P$ value for Hardy-Weinberg equilibrium (HWE), article quality. Different ethnicity descents were categorized as Asians or Caucasians. Study design was stratified into population-based and hospital-based studies. Any disagreement was resolved through discussion until the two reviewers reached a consensus.

\section{Quality score assessment}

Two reviewers (ZX Zhang and $\mathrm{X}$ Tong) independently assessed the quality of the included studies according to the Newcastle Ottawa Scale (NOS) (http:// www.ohri.ca/programs/clinical_epidemiology/oxford. asp). The scale consists of three components related to sample selection, comparability and ascertainment of exposure.

\section{Statistics analysis}

HWE was evaluated using Chi-square test in control groups of each study. Strength of association between HOTAIR SNPs and cancer risk was assessed by odds ratios (ORs) with 95\% confidence intervals (CIs). Statistical significance of the pooled OR was determined by $\mathrm{Z}$ test. Pooled ORs were used to calculate allele frequency comparison ( rs4759314: $\mathrm{G}$ versus A, rs920778: T versus C, rs7958904: C versus G, rs874945: A versus G,rs1899663: $T$ versus G). Five different ORs were calculated according to allele contrast model, dominant model, recessive model, heterozygote comparison and homozygote comparison. Subgroup analyses were performed by cancer types and ethnicities. Heterogeneity degree between different studies was determined by Q-statistic [44, 45]. If there was no significance in heterogeneity degree, the fixed effect model (Mantel-Haenszel method) would be used [46]. Otherwise, the random effect model (DerSimonian and
Laird method) would be used [47]. Heterogeneity effect was then quantified by $\mathrm{I}^{2}$ test. $\mathrm{I}^{2}$ statistics represented the proportion of variation across studies due to betweenstudy heterogeneity, and the values of $25 \%, 50 \%$ and $75 \%$ were regarded as cut-off points for low, moderate and high degrees of heterogeneity, respectively [44]. Sensitivity analysis was performed to evaluate the stability of the results. Publication bias was evaluated by funnel plot and quantified by Begg's test and Egger's test to assess funnel plot asymmetry $[48,49]$. Meta-analyses were performed with the software Stata (Version 12, College Station, Texas, USA). All statistical tests were two-sided and $p<0.05$ was considered statistically significant.

\section{ACKNOWLEDGMENTS}

Zhao-Xiong Zhang designed and wrote rough manuscript. Zhao-Xiong Zhang and Wan-Ni Zhang screened out relevant articles and extracted data. ZhaoXiong Zhang and Xue Tong analyzed data. Wei-Neng Fu carefully checked the results and revised the manuscript.

\section{CONFLICTS OF INTEREST}

All authors declare no conflicts of interest.

\section{GRANT SUPPORT}

This work was supported by two grants from the National Natural Science Foundations of China (81172577 and 81372876).

\section{REFERENCES}

1. Esteller M. Non-coding RNAs in human disease. Nat Rev Genet. 2011; 12:861-874.

2. Lee JT. Epigenetic regulation by long noncoding RNAs. Science. 2012; 338:1435-1439.

3. Kung JT, Colognori D, Lee JT. Long noncoding RNAs: past, present, and future. Genetics. 2013; 193:651-669.

4. Tran NT, Su H, Khodadadi-Jamayran A, Lin S, Zhang L, Zhou D, Pawlik KM, Townes TM, Chen Y, Mulloy JC, Zhao X. The AS-RBM15 lncRNA enhances RBM15 protein translation during megakaryocyte differentiation. Embo Rep. 2016; 17:887-900.

5. Yang F, Bi J, Xue X, Zheng L, Zhi K, Hua J, Fang G. Up-regulated long non-coding RNA H19 contributes to proliferation of gastric cancer cells. FEBS J. 2012; 279:3159-3165.

6. Pickard MR, Mourtada-Maarabouni M, Williams GT. Long non-coding RNA GAS5 regulates apoptosis in prostate cancer cell lines. Biochim Biophys Acta. 2013; 1832:1613-1623.

7. Ellis BC, Graham LD, Molloy PL. CRNDE, a long noncoding RNA responsive to insulin/IGF signaling, regulates 
genes involved in central metabolism. Biochim Biophys Acta. 2014; 1843:372-386.

8. Wang K, Liu CY, Zhou LY, Wang JX, Wang M, Zhao B, Zhao WK, Xu SJ, Fan LH, Zhang XJ, Feng C, Wang CQ, Zhao YF, et al. APF lncRNA regulates autophagy and myocardial infarction by targeting miR-188-3p. Nat Commun. 2015; 6:6779.

9. Guo H, Ahmed M, Zhang F, Yao CQ, Li S, Liang Y, Hua J, Soares F, Sun Y, Langstein J, Li Y, Poon C, Bailey SD, et al. Modulation of long noncoding RNAs by risk SNPs underlying genetic predispositions to prostate cancer. Nat Genet. 2016; 48:1142-1150.

10. Yuan H, Liu H, Liu Z, Owzar K, Han Y, Su L, Wei Y, Hung RJ, McLaughlin J, Brhane Y, Brennan P, Bickeboeller H, Rosenberger A, et al. A Novel Genetic Variant in Long Noncoding RNA Gene NEXN-AS1 is Associated with Risk of Lung Cancer. Sci Rep. 2016; 6:34234.

11. Yao L, Tak YG, Berman BP, Farnham PJ. Functional annotation of colon cancer risk SNPs. Nat Commun. 2014; 5:5114.

12. Cowper-Sal LR, Zhang X, Wright JB, Bailey SD, Cole MD, Eeckhoute J, Moore JH, Lupien M. Breast cancer riskassociated SNPs modulate the affinity of chromatin for FOXA1 and alter gene expression. Nat Genet. 2012; 44:1191-1198.

13. Myles S, Davison D, Barrett J, Stoneking M, Timpson N. Worldwide population differentiation at disease-associated SNPs. Bmc Med Genomics. 2008; 1:22.

14. Rinn JL, Kertesz M, Wang JK, Squazzo SL, Xu X, Brugmann SA, Goodnough LH, Helms JA, Farnham PJ, Segal E, Chang HY. Functional demarcation of active and silent chromatin domains in human HOX loci by noncoding RNAs. Cell. 2007; 129:1311-1323.

15. Chiyomaru T, Fukuhara S, Saini S, Majid S, Deng G, Shahryari V, Chang I, Tanaka Y, Enokida H, Nakagawa M, Dahiya R, Yamamura S. Long non-coding RNA HOTAIR is targeted and regulated by miR-141 in human cancer cells. J Biol Chem. 2014; 289:12550-12565.

16. Gupta RA, Shah N, Wang KC, Kim J, Horlings HM, Wong DJ, Tsai MC, Hung T, Argani P, Rinn JL, Wang Y, Brzoska P, Kong B, et al. Long non-coding RNA HOTAIR reprograms chromatin state to promote cancer metastasis. Nature. 2010; 464:1071-1076.

17. Kreile M, Piekuse L, Rots D, Dobele Z, Kovalova Z, Lace B. Analysis of possible genetic risk factors contributing to development of childhood acute lymphoblastic leukaemia in the Latvian population. Arch Med Sci. 2016; 12:479-485.

18. Grotenhuis AJ, Dudek AM, Verhaegh GW, Aben KK, Witjes JA, Kiemeney LA, Vermeulen SH. Independent Replication of Published Germline Polymorphisms Associated with Urinary Bladder Cancer Prognosis and Treatment Response. Bl Cancer. 2016; 2:77-89.

19. Murali A, Varghese BT, Kumar RR, Kannan S. Combination of genetic variants in cyclin D1 and retinoblastoma genes predict clinical outcome in oral cancer patients. Tumour Biol. 2016; 37:3609-3617.

20. Tian T, Li C, Xiao J, Shen Y, Lu Y, Jiang L, Zhuang X, Chu M. Quantitative Assessment of the Polymorphisms in the HOTAIR lncRNA and Cancer Risk: A Meta-Analysis of 8 Case-Control Studies. Plos One. 2016; 11:e152296.

21. Zhang J, Liu X, You LH, Zhou RZ. Significant association between long non-coding RNA HOTAIR polymorphisms and cancer susceptibility: a meta-analysis. Onco Targets Ther. 2016; 9:3335-3343.

22. Qi Q, Wang J, Huang B, Chen A, Li G, Li X, Wang J. Association of HOTAIR polymorphisms rs4759314 and rs920778 with cancer susceptibility on the basis of ethnicity and cancer type. Oncotarget. 2016; 7:38775-38784. doi: 10.18632/oncotarget.9608.

23. Zhang X, Zhou L, Fu G, Sun F, Shi J, Wei J, Lu C, Zhou C, Yuan Q, Yang M. The identification of an ESCC susceptibility SNP rs920778 that regulates the expression of lncRNA HOTAIR via a novel intronic enhancer. Carcinogenesis. 2014; 35:2062-2067.

24. Xue Y, Gu D, Ma G, Zhu L, Hua Q, Chu H, Tong N, Chen J, Zhang Z, Wang M. Genetic variants in lncRNA HOTAIR are associated with risk of colorectal cancer. Mutagenesis. 2015; 30:303-310.

25. Guo W, Dong Z, Bai Y, Guo Y, Shen S, Kuang G, Xu J. Associations between polymorphisms of HOTAIR and risk of gastric cardia adenocarcinoma in a population of north China. Tumour Biol. 2015; 36:2845-2854.

26. Bayram S, Sumbul AT, Batmaci CY, Genc A. Effect of HOTAIR rs920778 polymorphism on breast cancer susceptibility and clinicopathologic features in a Turkish population. Tumour Biol. 2015; 36:3863-3870.

27. Pan W, Liu L, Wei J, Ge Y, Zhang J, Chen H, Zhou L, Yuan Q, Zhou C, Yang M. A functional lncRNA HOTAIR genetic variant contributes to gastric cancer susceptibility. Mol Carcinog. 2016; 55:90-96.

28. Bayram S, Ulger Y, Sumbul AT, Kaya BY, Rencuzogullari A, Genc A, Sevgiler Y, Bozkurt O, Rencuzogullari E. A functional HOTAIR rs920778 polymorphism does not contributes to gastric cancer in a Turkish population: a case-control study. Fam Cancer. 2015; 14:561-567.

29. Du M, Wang W, Jin H, Wang Q, Ge Y, Lu J, Ma G, Chu H, Tong N, Zhu H, Wang M, Qiang F, Zhang Z. The association analysis of lncRNA HOTAIR genetic variants and gastric cancer risk in a Chinese population. Oncotarget. 2015; 6:31255-31262. doi: 10.18632/oncotarget.5158.

30. Yan R, Cao J, Song C, Chen Y, Wu Z, Wang K, Dai L. Polymorphisms in IncRNA HOTAIR and susceptibility to breast cancer in a Chinese population. Cancer Epidemiol. 2015; 39:978-985.

31. Zhou Q, Chen F, Fei Z, Zhao J, Liang Y, Pan W, Liu X and Zheng D. Genetic variants of IncRNA HOTAIR contribute to the risk of osteosarcoma. Oncotarget. 2016; 7:19928-34. doi: 10.18632/oncotarget.7957. 
32. Wu H, Shang X, Shi Y, Yang Z, Zhao J, Yang M, Li Y, Xu S. Genetic variants of lncRNA HOTAIR and risk of epithelial ovarian cancer among Chinese women. Oncotarget. 2016; 7:41047-41052. doi: 10.18632/oncotarget.8535.

33. Qiu H, Liu Q, Li J, Wang X, Wang Y, Yuan Z, Li J, Pei DS. Analysis of the association of HOTAIR single nucleotide polymorphism (rs920778) and risk of cervical cancer. APMIS. 2016.

34. Zhang C, Shen M, Xu X, Hu Y, Zhang Z, Duan H, Niu Y, Yuan H. Quantitative assessment of the association between functional long non-coding RNA HOTAIR genetic variants and cancer susceptibility. Int J Clin Exp Med. 2016; 9:2157-2165.

35. Palanichamy JK, Tran TM, Howard JM, Contreras JR, Fernando TR, Sterne-Weiler T, Katzman S, Toloue M, Yan W, Basso G, Pigazzi M, Sanford JR, Rao DS. RNAbinding protein IGF2BP3 targeting of oncogenic transcripts promotes hematopoietic progenitor proliferation. J Clin Invest. 2016; 126:1495-1511.

36. Shen CT, Qiu ZL, Song HJ, Wei WJ, Luo QY. miRNA-106a directly targeting RARB associates with the expression of $\mathrm{Na}(+) / \mathrm{I}(-)$ symporter in thyroid cancer by regulating MAPK signaling pathway. J Exp Clin Cancer Res. 2016; 35:101.

37. Ryan BM, Robles AI, McClary AC, Haznadar M, Bowman ED, Pine SR, Brown D, Khan M, Shiraishi K, Kohno T, Okayama H, Modali R, Yokota J, et al. Identification of a functional SNP in the 3'UTR of CXCR2 that is associated with reduced risk of lung cancer. Cancer Res. 2015; 75:566-575.

38. Ma G, Wang Q, Lv C, Qiang F, Hua Q, Chu H, Du M, Tong N, Jiang Y, Wang M, Zhang Z, Wang J, Gong W. The prognostic significance of HOTAIR for predicting clinical outcome in patients with digestive system tumors. J Cancer Res Clin Oncol. 2015; 141:2139-2145.

39. Li J, Wen W, Zhao S, Wang J, Chen J, Wang Y, Zhang Q. Prognostic role of HOTAIR in four estrogen-dependent malignant tumors: a meta-analysis. Onco Targets Ther. 2015; 8:1471-1482.

40. Zhang S, Chen S, Yang G, Gu F, Li M, Zhong B, Hu J, Hoffman A, Chen M. Long noncoding RNA HOTAIR as an independent prognostic marker in cancer: a meta-analysis. Plos One. 2014; 9:e105538.

41. Yan Y, Han J, Li Z, Yang H, Sui Y, Wang M. Elevated RNA expression of long noncoding HOTAIR promotes cell proliferation and predicts a poor prognosis in patients with diffuse large B cell lymphoma. Mol Med Rep. 2016; 13:5125-5131.

42. Shang C, Guo Y, Zhang H, Xue YX. Long noncoding RNA HOTAIR is a prognostic biomarker and inhibits chemosensitivity to doxorubicin in bladder transitional cell carcinoma. Cancer Chemother Pharmacol. 2016; 77:507-513.

43. Zhang YY, Huang SH, Zhou HR, Chen CJ, Tian LH, Shen JZ. Role of HOTAIR in the diagnosis and prognosis of acute leukemia. Oncol Rep. 2016.

44. Higgins JP, Thompson SG, Deeks JJ, Altman DG. Measuring inconsistency in meta-analyses. BMJ. 2003; 327:557-560.

45. Zintzaras E, Ioannidis JP. Heterogeneity testing in metaanalysis of genome searches. Genet Epidemiol. 2005; 28:123-137.

46. Mantel N, Haenszel W. Statistical aspects of the analysis of data from retrospective studies of disease. J Natl Cancer Inst. 1959; 22:719-748.

47. DerSimonian R, Laird N. Meta-analysis in clinical trials. Control Clin Trials. 1986; 7:177-188.

48. Begg CB, Mazumdar M. Operating characteristics of a rank correlation test for publication bias. Biometrics. 1994; 50:1088-1101.

49. Egger M, Davey SG, Schneider M, Minder C. Bias in metaanalysis detected by a simple, graphical test. BMJ. 1997; 315:629-634. 